\title{
Anterior lamellar recession in the management of the trachomatous cicatricial entropion of the upper eyelids: Outcomes and indications
}

\author{
Naser Owji, Mansooreh Jamshidian Tehrani \\ Poostchi Eye Research Center, Shiraz University of Medical Sciences, Shiraz, Iran
}

\begin{abstract}
Aim: To evaluate the success and complications of anterior lamellar recession (ALR) in trachomatous cicatricial entropion.

Methods: Twenty-six consecutive patients (forty upper eyelids) with trachomatous cicatricial entropion underwent ALR between 2003 and 2010. All patients had aberrant lashes with severe abnormal lid margin. Anatomical success was defined as disappearance of lid margin abnormality. Presence of aberrant lash did not indicate a treatment failure. Complete success was described as anatomical success without abrading abnormal lashes.

Results: ALR was performed on the 40 upper eyelids (19 right and 21 left). The average duration of follow-up time was 34 months (range: six to 84 months). Anatomical success was achieved in 28 out of 40 lids (70\%). Trichiasis developed in 18 eyelids post-operatively (45\%), so complete success rate was $55 \%$.

Conclusion: ALR is a well-established procedure with an acceptable success rate for management of trachomatous cicatricial entropion and severe lid margin abnormality. The most important drawback of this procedure is development of trichiasis.
\end{abstract}

Key words: Trachoma, cicatricial entropion, trichiasis, anterior lamellar recession (ALR)

\section{Introduction}

Cicatricial entropion results from a scarring and shortening of the conjunctival and tarsal plate. Various conditions such as auto-immune and inflammatory diseases, surgical and traumatic conditions, and infectious diseases may lead to cicatricial entropion. Trachoma is the most common infectious cause of blindness worldwide. ${ }^{1}$ Recurrent chronic conjunctivitis, most probably of the upper tarsal conjunctiva, leads to cicatricial entropion, abnormal eyelashes, and abnormal keratinized lid margin. Abrading eyelashes and keratinized lid margin cause mechanical trauma to the ocular surface and cornea leading to the patient's discomfort, foreign-body sensation, and decreased visual acuity secondary to corneal opacity. Early diagnosis of cicatricial eyelid changes and selection of an appropriate surgical procedure to create a healthy environment for the ocular surface play an important role in the prevention of blindness as a complication of trachoma.

ALR is one of the procedures used for correction of cicatricial entropion and trichiasis. $^{2-9}$ Few studies are available that present the effect of this procedure in trachomatous entropion and trichiasis. ${ }^{5,8,9}$ Because our country is an endemic area for trachoma, in the present study, we aimed to report the results of ALR in patients with trachomatous cicatricial entropion of the upper eyelid in our center. We also aimed to determine

Correspondence: Naser Owji, MD, Poostchi Eye Research Center, Poostchi Street, Shiraz, Iran. E-mail: owjin@sums.ac.ir,dr_oji@yahoo.com 
the indications of performing this procedure according to our experiences as well as reviewing the related studies.

\section{Patients and methods}

This study was performed on 40 upper eyelids of 26 patients with trachomatous cicatricial entropion who were operated by a single surgeon using ALR technique during 2003 to 2010. The protocol of the study was approved by the local Ethics Committee of the Poostchi Ophthalmology Research Center affiliated to Shiraz University of Medical Sciences, Shiraz, Iran. Informed consent was obtained from the patients after giving detailed information.

The inclusion criteria were presence of cicatricial entropion and aberrant lashes with sever abnormal lid margin. Eleven of 40 lids had had previous unsuccessful operations for entropion and abnormal lashes. Severe abnormal lid margin was defined as keratinized and rounding of the lid margin with posterior migration of meibomian glands orifices (Fig. 1).

Patients' demographic data, abnormal lids, extent of involvement (generalized or localized), tarsal plate consistency (existence of shrinkage, or loosening), and previous lid surgeries were evaluated by standard clinical history taking and detailed lid examination and inspection

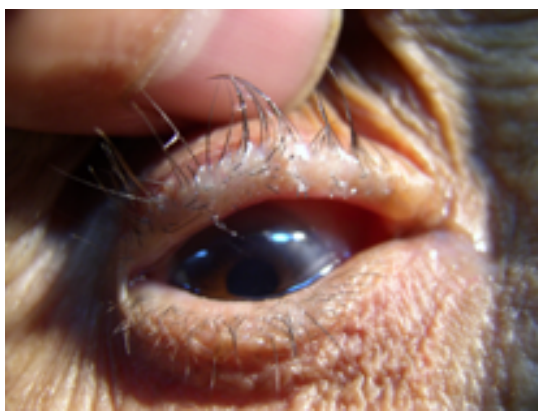

Fig. 1. Severe lid margin abnormality in patients with trachomatous entropion. before and after the surgery.

Extent of eyelid involvement was categorized as generalized (100\% of lid involvement) and localized eyelid involvement (less than $100 \%$ of lid involvement).

Tarsal plate consistency was categorized considering the presence or absence of tarsal shrinkage (shortening with or without thickening) or loosening (loss of tarsal consistency) or both.

\section{Surgical technique}

The operation was performed either under standby sedation or under general anesthesia, based on the patients' cooperation. Standby anesthesia was accomplished by intravenous sedation and local infiltration of $2 \%$ lidocaine through 1:100,000 epinephrine. In the general-anesthesia method, lidocaine injection was not administered.

For generalized involvement, the eyelid margin was incised along the most posterior abnormal eyelash from the lateral commissure to just lateral to the lacrimal punctum. For localized lid involvement, the incision was done two $\mathrm{mm}$ from the abnormal site with vertical relaxing incision of the skin, then the dissection was done toward the superior tarsal border and retractors released from tarsal plate. A ribbon of anterior lid margin including an abnormal row of lashes and keratinized tissue from anterior lamella, was excised. The skin and muscle flap along with the normal lashes of the entire extent of 
the eyelid were then recessed and fixed four $\mathrm{mm}$ superior to the margin of the upper eyelid, and anchored to the anterior tarsal surface using three or four 6-0 Vicryl horizontal mattress sutures (Fig. 2).

Post-operative care included prescribing a combination of a hydrocortisone eye ointment and erythromycin ointment for six weeks, which were tapered until discontinued. The patients were visited in regular intervals of one day, one week, one month, three months, and six months post-operatively and after that at the discretion

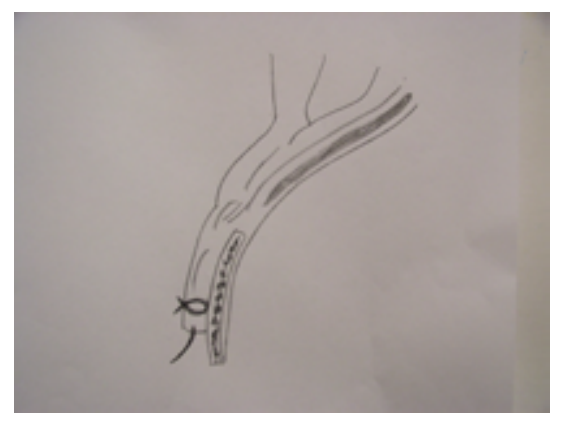

Fig. 2. Anterior lamellar recession procedure. of the surgeon.

The success of the operations was categorized as anatomical or complete. Anatomical success was defined as disappearance of lid margin abnormality and keratinization, appropriate localization of mucocutaneous junction, and no skin touching the ocular surface. Presence of aberrant lash did not indicate a treatment failure. Complete success was described as anatomical success without abrading the abnormal lashes.

Statistical analysis was performed using Statistical Package for Social Sciences software version 17 (SPSS Inc., Chicago, Illinois). For subgroup analyses, nominal variables were analyzed using Chi-Square test. A p value $\leq 0.05$ was considered statistically significant.

\section{Results}

Twenty-six patients (40 upper eyelids) including seven men and 19 women were diagnosed to have trachomatous trichiasis. Generalized involvement was present in 25 eyelids and localized involvement was present in 15 eyelids. The age range was from 51 to 90 years (mean: 68.8 years).

Of the 40 eyelids, 19 had good tarsal consistency, six had loosening, four had shrinkage, and 11 had both tarsal loosening and shrinkage. ALR was performed for 19 right and 21 left eyelids. The average duration of the follow-up visits was 34 months (range: 6 to 84 months). Previous operations were done on 11 of the 40 eyelids.

Anatomical success was achieved in 28 lids (70\%). Trichiasis developed in 18 (45\%) eyelids post-operatively, so the complete success rate was $55 \%$ in our series.

Nineteen patients had good tarsal consistency (47.5\%) and 21 (52.5\%) patients had abnormal tarsal consistency. The success in the good tarsal group was 15 out of 19 lids (78.9\%) and in the abnormal tarsus group 13 out of 21 lids (61.9\%). Fischer exact test did not show a significant association between tarsal consistency and final success, which could be caused by low power of the study $(P=0.5)$. Only a subgroup analysis between the success of the good-tarsus group and the group with shrinkage and loosening showed a significant difference (15 of 19 [78.9\%] versus 3 of 11 [27.2\%]. [P=0.008] The success in the group with generalized lid involvement was 17 of 25 lids $(68 \%)$ and in the group with localized lid involvement 11 of 15 lids (73.3\%). Chi square test did not show a significant association between the extent of lid involvement and primary and final success, which can be explained again by the low power of the study $(P=0.5)$. 
No significant post-operative complications, such as hematoma or suture abscess, were observed.

\section{Discussion}

ALR is a well-established procedure for the correction of cicatricial entropion and trichiasis. This procedure is performed using various techniques. The procedure has been presented in the literature with different terms including: grey line split with anterior lamellar reposition; $;, 4,7$ tarso-conjunctival advancement; ${ }^{3}$ lamellar division; ${ }^{2,5}$ tarsal advance; ${ }^{9}$ and ALR. ${ }^{7}$ The ALR procedure involves complete splitting of the lid from the grey line or posterior to the more posterior aberrant eyelashes and keratinized lid margin with or without disinsertion of eyelid retractors and subsequent recession of anterior lamella three to seven $\mathrm{mm}$ posterior to the lid margin (Fig. 2).

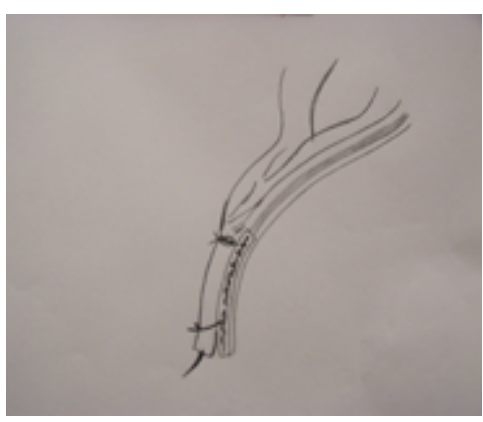

Fig. 3. Anterior lamellar reposition and lid splitting procedure.

ALR should be differentiated from anterior lamellar repositioning and lid split. Some investigators used the term 'anterior lamellar repositioning' for the presentation of an ALR procedure, ${ }^{4,6,7}$ and erroneously the outcomes of these two procedures have been compared, ${ }^{7}$ while the technique, outcome, and indications of the two procedures are different. ${ }^{2}$ It concerns anterior lamellar repositioning via lid crease incision and one-to-two-mm lid margin splitting without ALR (Fig. 3).

ALR is used for correction of entropion ${ }^{2-7}$ and trichiasis. ${ }^{8,9}$ This procedure was investigated in the treatment of trachomatous trichiasis in some articles. ${ }^{9}$ In some studies, the major cause of entropion and trichiasis was trachoma.,8 To the best of our knowledge our study is the first report showing the outcome of ALR in trachomatous cicatricial entropion of the upper eyelid with long-term follow-up. We performed this procedure in patients with severe cicatricial lid margin abnormality secondary to trachoma. The anatomical success was $70 \%$ and complete success rate was $55 \%$.The success rate was related to tarsal-plate consistency. In patients with normal tarsus, the ALR success rate was $78.9 \%$. The success rate in patients with tarsal shrinkage and loosening was $27.2 \%$. The difference was statically significant $(P=0.008)$.

Kemp and Collin ${ }^{2}$ recommended this procedure for patients with moderate entropion and marked lid retraction and for patients with severe entropion. Sodhi and Pandey ${ }^{6}$ used ALR in 84 eyelids with cicatricial entropion and various etiologies with an overall success rate of $88.09 \%$ after one year follow up. The severity of entropion was not mentioned. ALR was performed by Pungnumkul ${ }^{8}$ on 18 eyelids with major upper lid trichiasis (> five eyelashes) with a success rate of $77.77 \%$ and a mean follow up of 144 days. Reacher et al. ${ }^{9}$ used ALR in patients with major trichiasis. The overall success rate in 41 patients was $27 \%$. The severity of entropion was not determined in this study.

Koreen et al.' used ALR with buccal mucousal grafting for the correction of 35 eyelids with moderate to severe cicatricial entropion. Trichiasis was present in $94 \%$ of the eyelids pre-operatively. The anatomical success rate of the primary repair was $77 \%$. Trichiasis or 
distichiasis were present in $54 \%$ of the eyelids post-operatively.

Direct comparison of these studies with ours is not possible. The wide range of the success rate for ALR procedures (27\%-97\%) reported by several studies could be due to different population, different types of eyelid abnormalities (trichiasis and/or entropion) included in these studies, different severity of entropion or trichiasis, different surgical skills, different techniques and follow-up durations, and difference in the definition of success.

One of the most important factors affecting the success of a specific procedure for the correction of cicatricial entropion and associated eyelid abnormalities is the selection of the appropriate surgery. The severity of entropion and the association of misdirected eyelashes and severity of lid margin abnormality should be considered for the selection of the appropriate surgical procedure. ${ }^{10}$ The severity of cicatricial entropion could be classified as mild, moderate, and severe. Mild entropion is defined as a condition in which the eyelashes do not abrade the cornea in primary position but touch to the globe in up-gaze or down-gaze. Moderate entropion is diagnosed when there is a lash globe contact in the primary position. Severe entropion is manifested as a totally disorganized margin with metaplastic lashes and trichiasis, definite or gross lid retraction, and keratin plaques on the palpebral conjunctival surface. ${ }^{2}$ For mild to moderate cicatricial entropion without lid retraction, anterior lamellar repositioning with or without lid splitting, Wies procedure, and tarsal fracture might be the procedures of choice., ${ }^{2,10}$ In moderate cicatricial entropion with lid retraction and severe entropion, ALR could be used. ${ }^{2}$ We used this procedure in patients with severe lid margin abnormality irrespective of severity of entropion to keep away keratinized tissue and abnormal lashes from the ocular surface.

Several surgeons modified the ALR technique to improve the success rate. Some of them used an anchoring suture in the fornix that stably repositioned the anterior lamella on the posterior lamella., ${ }^{4,5}$ Fixing the tarsus to the cheek by tarsal traction sutures has been reported. ${ }^{3}$ Covering of the bare tarsus by an amniotic membrane graft and a mucous membrane graft were performed by others. ${ }^{4,7}$

We did not use these modified techniques. The shortcoming of the ALR procedure in our study was the development of trichiasis. In $45 \%$ of our patients, abrading lashes were present post-operatively. This is compatible with the Koreen et al. study. In their patients, trichiasis and distichiasis were present in $54 \%$ of eyelids postoperatively after ALR and mucous membrane graft. It seems that coverage of the bare tarsus with a mucous membrane graft did not effectively prevent recurrence of aberrant lashes.

We agree with Sodhi ${ }^{6}$ that recurrence of trichiatic lashes is due to gradual downward sliding of recessed anterior lamella, so placement of a fornix suture and tarsal traction sutures may stable the anterior lamella in a recessed position and improve the success of ALR (Fig. 4) As previously mentioned, with this modified techniques the success rate (no

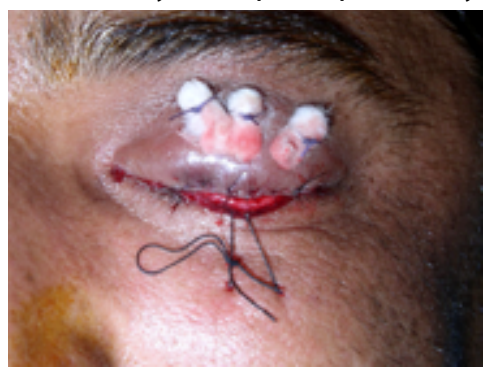

Fig. 4. Anterior lamellar recession with fornix suture and fixing traction suture. 
lash abrading the globe) of this procedure reported by Sodhi ${ }^{6}$ and Kuckelkorn ${ }^{3}$ was $88.09 \%$ and $80.55 \%$, respectively. The other shortcomings of this procedure are that it is a difficult technique for not so skillful surgeons, the prolonged surgical time, and disturbed cosmesis at early post-operative days.

In spite of the above-mentioned shortcomings, ALR has several advantages, including:

- In ALR, keratinized lid margin could be trimmed and new, healthy, smooth lid margin is formed;

- Aberrant lashes could be excised or recessed away from the lid margin;

- Release of eyelid retractors may improve the lid retraction caused by the cicatricial process or previous failed surgery.This procedure does not involve the conjunctiva directly, so it does not aggravate auto-immune conjunctival diseases.

We can summarize the indications of ALR as follows:

- Severe lid margin abnormality with keratinized lid margin and aberrant lashes; ${ }^{2,6,9}$

- Presence of major trichiasis ( $>$ five eyelashes);, 8,9

- Moderate to severe entropion with lid retraction, ${ }^{2,6}$

- Entropion and trichiasis in auto-immune conjunctival diseases, where conjunctival incision may aggravate the disease.

\section{Conclusion}

ALR is recommended and used for correction of cicatricial entropion of all severities, and for treatment of trichiasis with acceptable success rate. This procedure addresses all cicatricial eyelid changes secondary to trachoma, but we recommend this procedure in selected cases with significant cicatricial eyelid margin abnormalities and trichiasis, and in cases with auto-immune conjunctival diseases. For a lesser degree of cicatricial eyelid changes, simpler procedures should be used.

\section{References}

1. Burton MJ. Trachoma: an overview. Br Med Bull 2007;84:99-116. Epub 2008 Jan 5.

2. Kemp EG, Collin JR. Surgical management of upper lid entropion. Br J Ophthalmol 1986;70(8):575-579.

3. Kuckelkorn R, Schrage N, Becker J, Reim M. Tarsoconjunctival advancement: a modified surgical technique to correct cicatricial entropion and metaplasia of the marginal tarsus. Ophthalmic Surg Lasers 1997;28(2):156-161.

4. Seng-Ei Ti, Sharon LC, Tow Soon-Phaik Chee. Amniotic Membrane Transplantation in Entropion surgery. Ophthalmolgy 2001;108(7):1209-1217.

5. Sodhi PK, Yadava U, Mehta DK. Efficacy of lamellar division for correcting cicatricial lid entropion and its associated features unrectified by the tarsal fracture technique. Orbit 2002;21(1):9-17.

6. Sodhi PK, Yadava U, Pandey RM, Mehta DK. Modified grey line split with anterior lamellar repositioning for treatment of cicatricial lid entropion. Ophthalmic Surg Lasers. 2002;33(2):169-174.

7. Koreen IV, Taich A, Elner VM. Anterior lamellar recession with buccal mucous membrane grafting for cicatricial entropion. Ophthal Plast Reconstr Surg 2009;25(3):180-184.

8. Paichit Pongnumkul. Modified Grey Line Split with Anterior Lamellar Repositioning for Upper Lid Trichiasis. Thai J Ophthalmol 2003;17(2):153-161.

9. Reacher $\mathrm{MH}$, Huber MJ, Canagaratnam R, Alghassany A. A trial of surgery for trichiasis of the upper lid from trachoma. Br J Ophthalmol 1990;74(2):109-113.

10. Owji N, Bagheri A, Aslani A. Combined Wies procedure and direct internal eyelash bulb extirpation an effective procedure for treatment of cicatricial entropion and trichiasis. Asian J Opthalmol 2006:8(1):28-30. 